\title{
Conversion surgery for metastatic gastric cancer at 2 years after initial diagnosis of cancer of unknown primary with metastasis of cervical lymph nodes and ovary: a case report
}

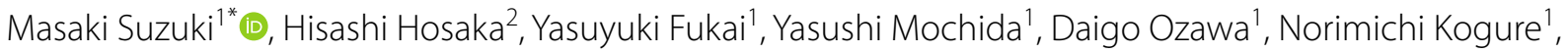
Kazunosuke Yamada ${ }^{1}$ and Hitoshi Ojima

\begin{abstract}
Background: Patients with stage IV gastric cancer have a poor prognosis despite improvements in intensive treatment regimens, including chemotherapy. Recently, conversion surgery has received much attention as it can provide long-term survival in stage IV gastric cancer patients who are responsive to chemotherapy. Herein, we describe the case of a patient who underwent conversion surgery for metastatic gastric cancer that was performed over 2 years after an initial diagnosis of cancer of unknown primary (CUP) with metastasis of the cervical lymph nodes and the ovary.
\end{abstract}

Case presentation: A 67-year-old woman with cervical lymphadenopathy was referred to our hospital. Computed tomography showed left cervical lymphadenopathy and bilateral ovarian enlargement. Endoscopic survey revealed no signs of malignancy in the upper or the lower gastrointestinal tract. Pathological findings after cervical lymphadenectomy revealed a signet-ring cell carcinoma and were suggestive of gastric cancer metastases. However, multiple evaluations yielded no evidence of gastric cancer and the patient was diagnosed with CUP. She was prescribed chemotherapy for gastric cancer and underwent bilateral oophorectomy after undergoing chemotherapy for 18 months. Pathologic analysis of oophorectomy tissue revealed findings identical to those seen in the cervical lymph nodes. At about 2 years after the initial diagnosis, an esophagogastroduodenoscopy revealed evidence of gastric cancer. We performed a distal gastrectomy with D2 lymphadenectomy. Her postoperative course was uneventful and she remains alive with no signs of disease recurrence at 3 months post-surgery.

Conclusions: To the best of our knowledge, this is the first report describing successful conversion surgery for stage IV gastric cancer in a patient whose cancer was definitively diagnosed 2 years after an initial diagnosis of CUP.

Keywords: Gastric cancer, Cancer of unknown primary, Conversion surgery, Distal gastrectomy, Cervical lymph node metastases, Ovarian metastases, Krukenberg tumor

\footnotetext{
*Correspondence: masakick11@gmail.com

${ }^{1}$ Department of Gastroenterological Surgery, Gunma Prefectural Cancer

Center, 617-1, Nishimach, Oota, Gunma 373-0828, Japan

Full list of author information is available at the end of the article
}

\begin{abstract}
Background
Despite early diagnosis and improved intensive treatments, gastric cancer remains a leading cause of malignancy-related death worldwide [1]. Most gastric cancer patients are not eligible for radical surgery due to the presence of locally advanced or metastatic disease [2]. With the development of multiple therapeutic approaches, standard treatment for gastric cancer is
\end{abstract}


described in the Japanese treatment guidelines for gastric cancer [3]. Recently, conversion surgery has emerged as a promising strategy that can provide long-term survival in patients with stage IV gastric cancer who are responsive to chemotherapy [4].

Although there are a few case reports on conversion surgery for gastric cancer [5-7], none describe a case that was initially diagnosed as cancer of unknown primary (CUP). Here we report a case of successful conversion surgery that was performed after a cervical lymphadenectomy and a bilateral oophorectomy, wherein definitive diagnosis of gastric cancer could only be established about 2 years after an initial diagnosis of CUP.

\section{Case presentation}

A 67-year-old woman with left cervical lymphadenopathy was referred to our hospital for further evaluation following a diagnosis of signet-ring cell carcinoma after a needle biopsy at a local medical doctor. The patient had an Eastern Cooperative Oncology Group Performance Status of 0. Her serum carbohydrate antigen (CA) 19-9 and carcinoembryonic antigen (CEA) levels were $16.8 \mathrm{U} /$ $\mathrm{ml}$ and $2.5 \mathrm{ng} / \mathrm{ml}$, respectively. Ultrasonography revealed three instances of lymph node enlargement in the left cervical area (Fig. 1a). Computed tomography (CT) showed left cervical lymphadenopathy and bilateral ovarian enlargement (Fig. 2a), but without specific signs of gastric cancer or lymph node metastasis surrounding the stomach. Esophagogastroduodenoscopy (EGD) revealed only slight atrophy of the stomach and there were no findings that indicated gastric cancer. Colonoscopy was also unremarkable. To make a definite diagnosis, we performed a lymphadenectomy of the left cervical region and pathological analysis revealed the presence of signetring cells and a poorly differentiated adenocarcinoma, which were suggestive of metastases originating from gastric cancer (Fig. 1b). However, fluorodeoxyglucose (FDG) positron emission computed tomography (PETCT) showed no abnormal uptake, and although we could not detect a primary lesion at that point, we recommended chemotherapy pertinent to gastric cancer.

The patient received monotherapy with oral S-1 ( $100 \mathrm{mg} /$ body/day) for the first 4 weeks of a 6-week cycle, and after three courses of chemotherapy, CT showed a reduction in ovarian metastases without the appearance of new lesions; however, EGD continued to reveal no signs of gastric cancer. Although treatment with S-1 was effective, the patient complained of general fatigue, which was accompanied by an elevation in liver enzymes, and she was diagnosed as being allergic to S-1. Her chemotherapy regimen was switched to nab-paclitaxel (nab-PTX), which consisted of a 4-week course of intravenous nab-PTX (100 mg/body) on days 1,8 , and 15 . While continuing this regimen for 18 months, the bilateral ovarian metastases remained stable. As there was no evidence of other lesions, including in the stomach, we performed a bilateral oophorectomy. There were no remarkable changes in gastric serosa and surrounding tissues of stomach and microscopic examination of the specimen confirmed a diagnosis of metastatic adenocarcinoma that consisted of a signet-ring cell carcinoma and a poorly differentiated adenocarcinoma (Fig. $2 \mathrm{~b}$ ). These
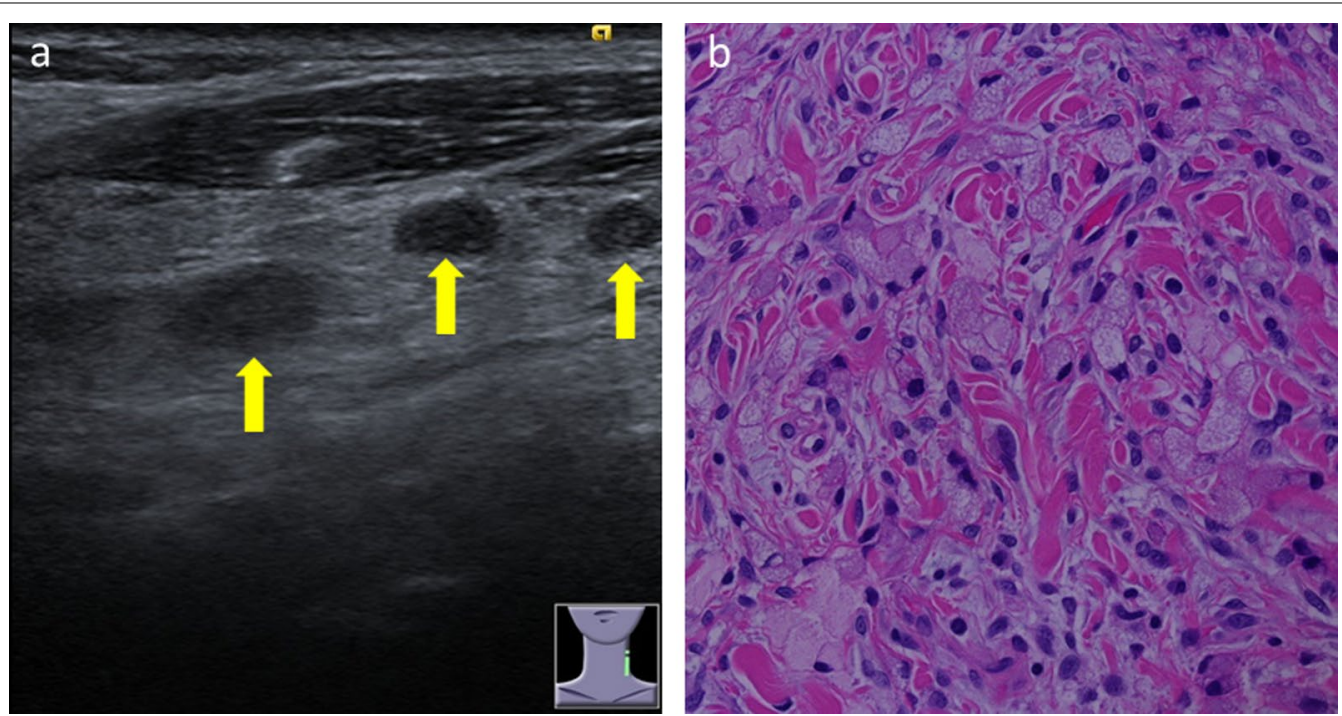

Fig. 1 Ultrasonography and pathological findings in cervical lymph nodes. a Ultrasonography showed three pieces of lymph node enlargement in left cervical area (arrow). b Pathological findings revealed signet-ring cell and a poorly differentiated adenocarcinoma ( $\times 400$ magnification) 

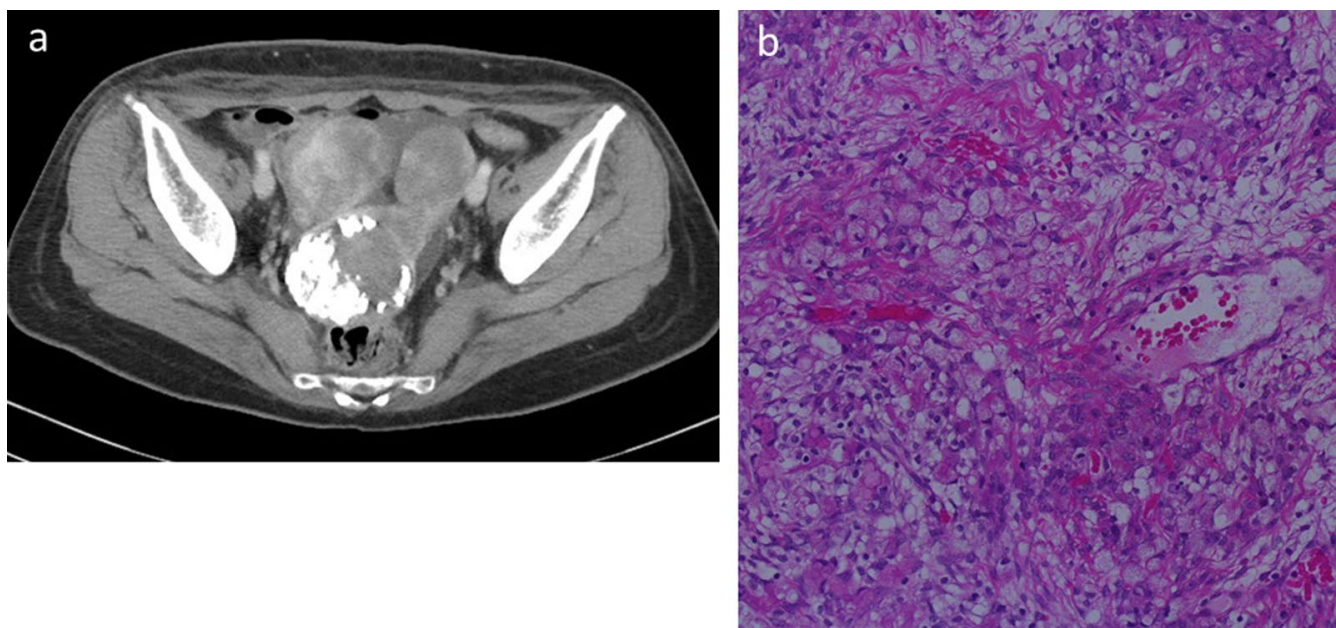

Fig. 2 Computed tomography (CT) and pathological findings in the ovary. a Abdominal CT showed bilateral ovarian enlargement with uterine calcification due to myoma. b Pathological findings revealed signet-ring cells and a poorly differentiated adenocarcinoma ( $\times 400$ magnification)

findings again suggested the presence of a primary gastric lesion.

The patient was carefully followed up with continued chemotherapy (nab-PTX). At 3 months after the oophorectomy, we detected a limited rough-surfaced mucosa with slight redness near the pyloric ring that stained positive for indigo carmine during endoscopic examination without any abnormality in other areas of antrum and gastric body (Fig. 3a). Biopsy specimens revealed a poorly differentiated adenocarcinoma with signet-ring cells (Fig. 3b), and this was considered as evidence of gastric cancer. Nonetheless, CT showed no specific changes in the stomach or in the nearby lymph nodes and PET-CT also showed no abnormal uptake in the whole body. We discussed the possibility of a R0 resection and decided to perform conversion surgery. We discussed the method of
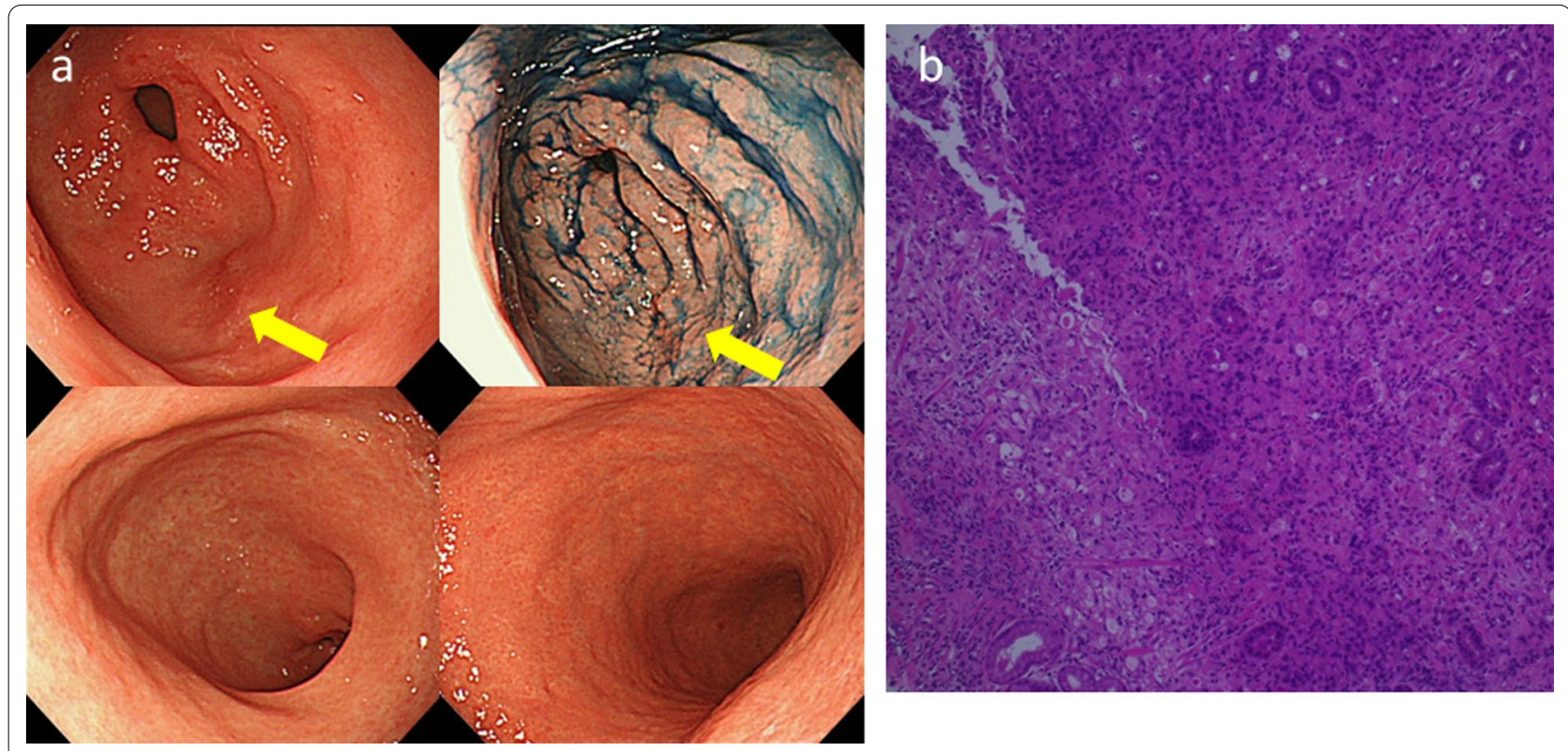

Fig. 3 Esophagogastroduodenoscopy and pathological findings in the stomach. a Esophagogastroduodenoscopy showed limited rough-surfaced mucosa with slight redness changes near the pyloric ring (arrow) that stained positive for indigo carmine staining (arrow). There were no specific signs in other areas of antrum and gastric body. b Biopsy specimens revealed a poorly differentiated adenocarcinoma with signet-ring cells $(\times 200$ magnification) 
surgery, and decided to proceed with distal gastrectomy considering the postoperative nutrition and absence of obvious signs that indicate the extent of the cancer in the upper area.

Thus, a definitive diagnosis could be established only about 2 years after the initial diagnosis of CUP and the patient underwent a distal gastrectomy with D2 lymphadenectomy. Detecting the cancerous area based on macroscopic findings was challenging (Fig. 4) and we did not find lymph node enlargement or peritoneal metastases intraoperatively. Pathological evaluation was graded as type 5 with T3 invasion that consisted of a poorly differentiated adenocarcinoma with signet-ring cells. The histological response of the primary and lymphatic tumor was grade 1a. Although the cancerous tissue was spread over a wide area with multiple lymph node metastases (27/32) (N3b), unexpectedly, both proximal and distal margins and the peritoneal washing were negative for cytology, according to the Japanese Classification of Gastric Carcinoma [3]. Her postoperative course was uneventful, and the patient was discharged on postoperative day 12. Currently, the patient is alive with no sign of disease recurrence at 3 months post-surgery.

\section{Conclusions}

We describe a case of metastatic gastric cancer that could be successfully treated with chemotherapy and conversion surgery. To the best of our knowledge, this is the first report of stage IV gastric cancer being managed by conversion surgery, wherein the initial diagnosis was CUP and a definitive diagnosis could only be established 2 years later.
CUP is defined as histologically confirmed metastatic tumors whose primary site cannot be identified upon standard pretreatment evaluation [8]. CUP occurs roughly equally in men and women, with an average ageat-diagnosis of 60 years, and accounts for $3 \%$ to $5 \%$ of all tumors [8]. It has been reported that a poorly differentiated adenocarcinoma infiltrates the submucosal layer without destroying the structure of the stomach wall, which is called a type of gastric linitis plastica [9]. Macroscopically, this is characterized by a diffuse thickening of the gastric wall, and microscopically, by the existence of poorly differentiated adenocarcinoma or signet-ring cells. Specimens obtained from a standard endoscopic biopsy rarely provide confirmative diagnosis because lesions in the submucosa are difficult to reach using forceps; therefore, other reported options used to obtain samples for a definite diagnosis include endoscopic ultrasound or the endoscopic submucosal dissection technique [10, 11]. Moreover, PET-CT, which is valuable for making diagnoses in many cancers, has been reported to be a poor detector of gastric cancer, especially signet-ring cell carcinoma [12], because signet-ring cell carcinoma are rarely positive for glucose transporter 1 expression, which is related to cellular FDG uptake. In the present case, we could not initially detect any evidence of gastric cancer and methods that can identify such cancers in their early stages must be explored. Nonetheless, we suspected the possibility of gastric cancer based on pathological findings of the biopsied cervical lymph nodes and the bilateral ovarian metastases, which pointed to gastric origins of the cancer. Thus, we had appropriately prescribed gastric cancer chemotherapy in our patient.

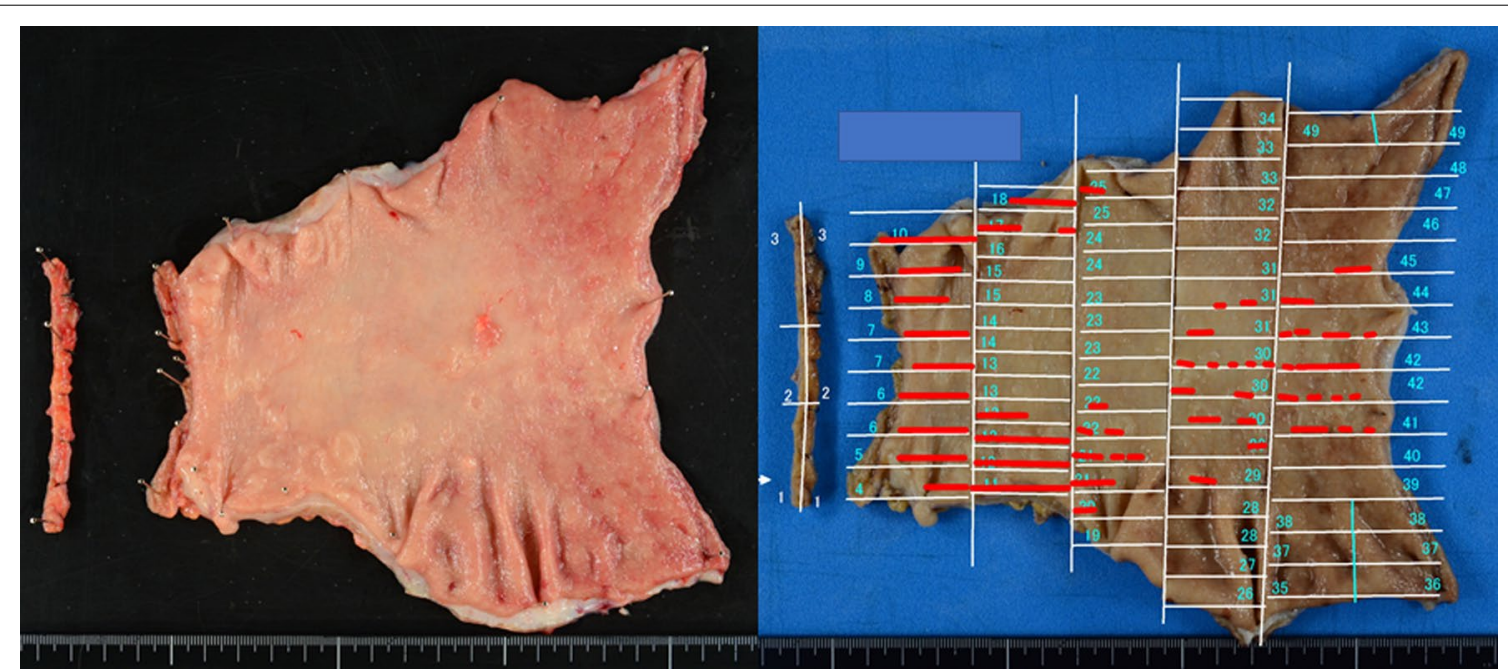

Fig. 4 Macroscopic findings in the resected stomach. Cancer cells were seen only at the range of the red lines with no involvement of the proximal or distal margins 
Krukenberg tumor $(\mathrm{KT})$ is a malignancy that has metastasized to the ovaries, and it is well known that gastric cancer is the leading primary lesion for KT [13]. Previous studies have shown that the prognosis in KT is poor, with median survival ranging from 9 to 11 months [14]. No optimal treatment strategies or guidelines have been established for patients diagnosed with KT of gastric origin, and even though recent studies have reported on the efficacy of oophorectomy, a survival benefit due to the procedure remains controversial $[15,16]$. In our patient, as we did not detect any metastases other than those in the ovary for a long time after initiating chemotherapy, we concluded that the lesion was limited to the ovary and that we could control the disease by performing an oophorectomy.

Conversion surgery for gastric cancer has emerged as a promising therapeutic tool. It is defined as surgical treatment aimed at achieving an $\mathrm{R} 0$ resection after chemotherapy for tumors that were originally unresectable or only marginally resectable due to technical and/ or oncological reasons [17]. In colorectal cancer patients, a surgical approach to metastatic lesions has played a crucial role in prolonging survival $[18,19]$. In contrast, the definition of conversion therapy and the indications for the procedure are yet to be clarified in patients with gastric cancer. Nevertheless, Yoshida et al. have proposed a system to help clarify these indications for conversion therapy [20] wherein they divided stage IV gastric cancer patients into four categories based on the biology and the heterogeneity of the tumors. Importantly, they have specifically highlighted the impact of the existence of macroscopic peritoneal dissemination. In our case, lymphatic metastases were limited to the left cervical region and distant metastases were only the KT, i.e., no metastasis to the liver or lung. Although classically it was thought that direct seeding across the abdominal cavity accounted for the spread of KT, lymphatic dissemination has also been demonstrated [21]. In fact, our patient had no signs of peritoneal dissemination as her peritoneal washing cytology grade was CY0. Therefore, the cancer in our patient could be classified as category 2, which is defined by Yoshida et al. as the most responsive to conversion surgery [20].

Recently, treatment choices in systemic chemotherapy have increased significantly, such as molecular-targeted drugs and immune checkpoint inhibitors [22-24]. The importance of conversion surgery, which consists of a combination of chemotherapy and surgical treatment, is expected to grow in the future. Although we could not detect the primary lesion in our patient for about 2 years, careful follow-up can indicate the need for surgical resection without delay. A proper survey is indispensable in treatment regimens that aim to provide a good prognosis.
We successfully used R0 surgery in our patient, which, according to the Japanese Classification of Gastric Carcinoma, is defined by the presence or absence of residual tumor after surgery [3]. However, poorly differentiated adenocarcinoma is known to have a poor prognosis [25] and our patient had multiple lymph node metastases. Therefore, careful and continuous follow-up is required.

In summary, we describe the case of a patient who underwent conversion surgery for stage IV metastatic gastric cancer, which could only be definitively diagnosed about 2 years after an initial diagnosis of CUP with metastasis of the cervical lymph nodes and the ovary. We suggest that conversion surgery, while challenging, is a promising treatment strategy for stage IV gastric cancer.

\section{Abbreviations}

CUP: Cancer of unknown primary; CT: Computed tomography; EGD: Esophagogastroduodenoscopy; FDG: Fluorodeoxyglucose; PET-CT: Positron emission computed tomography; nab-PTX: Nab-paclitaxel; KT: Krukenberg tumor.

\section{Acknowledgements \\ The authors would like to thank Enago (www.enago.jp) for the English language review.}

\section{Authors' contributions}

$\mathrm{MS}$ reported the case and wrote the manuscript. $\mathrm{HH}$ contributed to performing the chemotherapy. YF, YM, DO, NK, KY, and $\mathrm{HO}$ participated in the surgeries. All authors read and approved the final manuscript.

\section{Funding}

No grant support or funding from public institutions or private enterprises was received for this case report.

\section{Availability of data and materials}

The data supporting the conclusions of this article are included within the article.

\section{Declarations}

Ethics approval and consent to participate

Not applicable.

\section{Consent for publication}

Written informed consent was obtained from the patient for publication of this case report and accompanying images.

\section{Competing interests}

The authors declare that they have no competing interests.

\section{Author details}

${ }^{1}$ Department of Gastroenterological Surgery, Gunma Prefectural Cancer Center, 617-1, Nishimach, Oota, Gunma 373-0828, Japan. ${ }^{2}$ Department of Gastroenterology, Gunma Prefectural Cancer Center, Oota, Gunma, Japan.

Received: 10 January 2021 Accepted: 24 February 2021

Published online: 04 March 2021

\section{References}

1. Ferro A, Peleteiro B, Malvezzi M, Bosetti C, Bertuccio P, Levi F, et al. Worldwide trends in gastric cancer mortality (1980-2011), with predictions to 2015, and incidence by subtype. Eur J Cancer. 2014;50:1330-44 
2. Cervantes A, Roselló S, Roda D, Rodríguez-Braun E. The treatment of advanced gastric cancer: current strategies and future perspectives. Ann Oncol. 2008;19(Suppl 5):v103-7.

3. Japanese gastric cancer treatment guidelines 2018 (5th edition). Gastric Cancer. 2020; https://doi.org/10.1007/s10120-020-01042-y

4. Beom SH, Choi YY, Baek SE, Li SX, Lim JS, Son T, et al. Multidisciplinary treatment for patients with stage IV gastric cancer: the role of conversion surgery following chemotherapy. BMC Cancer. 2018;18:1116.

5. Serizawa A, Taniguchi K, Yamada T, Amano K, Kotake S, Ito S, et al. Successful conversion surgery for unresectable gastric cancer with giant para-aortic lymph node metastasis after downsizing chemotherapy with S-1 and oxaliplatin: a case report. Surg Case Rep. 2018;4:88.

6. Namikawa T, Tsuda S, Fujisawa K, Iwabu J, Uemura S, Tsujii S, et al. Conversion surgery after S-1 plus oxaliplatin combination chemotherapy for advanced gastric cancer with multiple liver metastases. Clin J Gastroenterol. 2018;11:297-301.

7. Matsumoto R, Arigami T, Matsushita D, Okubo K, Tanaka T, Yanagita S, et al. Conversion surgery for stage IV gastric cancer with a complete pathological response to nivolumab: a case report. World J Surg Oncol. 2020;18:179.

8. Losa F, Soler G, Casado A, Estival A, Fernández I, Giménez S, et al. SEOM clinical guideline on unknown primary cancer (2017). Clin Transl Oncol. 2018;20:89-96.

9. Kodera Y, Nakanishi H, Ito S, Mochizuki Y, Yamamura Y, Fujiwara M, et al. Detection of disseminated cancer cells in linitis plastica-type gastric carcinoma. Jpn J Clin Oncol. 2004;34:525-31.

10. Zhou XX, Pan HH, Usman A, Ji F, Jin X, Zhong WX, et al. Endoscopic ultrasound-guided deep and large biopsy for diagnosis of gastric infiltrating tumors with negative malignant endoscopy biopsies. World J Gastroenterol. 2015;21:3607-13.

11. Chiyo T, Kobara H, Mori H, Katsuki N, Haba R, Masaki T. Submucosal endoscopic sampling for indefinite gastric linitis plastica infiltrating into the submucosal layer. J Gastrointestin Liver Dis. 2015;24:375-8.

12. Shimada H, Okazumi S, Koyama M, Murakami K. Japanese Gastric Cancer Association Task Force for Research Promotion: clinical utility of ${ }^{18} \mathrm{~F}$-fluoro-2-deoxyglucose positron emission tomography in gastric cancer. A systematic review of the literature. Gastric Cancer. 2011;14:13-21.

13. McGill F, Ritter DB, Rickard C, Kaleya RN, Wadler S, Greston WM. Management of Krukenberg tumors: an 11-year experience and review of the literature. Prim Care Update Ob Gyns. 1998;5:157-8.

14. Kiyokawa T, Young RH, Scully RE. Krukenberg tumors of the ovary: a clinicopathologic analysis of 120 cases with emphasis on their variable pathologic manifestations. Am J Surg Pathol. 2006;30:277-99.

15. Rosa F, Marrelli D, Morgagni P, Cipollari C, Vittimberga G, Framarini M, et al. Krukenberg tumors of gastric origin: the rationale of surgical resection and perioperative treatments in a multicenter western experience. World J Surg. 2016;40:921-8.

16. Ma F, Li Y, Li W, Kang W, Liu H, Ma S, et al. Metastasectomy improves the survival of gastric cancer patients with Krukenberg tumors: a retrospective analysis of 182 patients. Cancer Manage Res. 2019;11:10573-80

17. Suzuki T, Tanabe K, Taomoto J, Yamamoto H, Tokumoto N, Yoshida K, et al. Preliminary trial of adjuvant surgery for advanced gastric cancer. Oncol Lett. 2010;1:743-7.

18. Adam R, Wicherts DA, de Haas RJ, Ciacio O, Lévi F, Paule B, et al. Patients with initially unresectable colorectal liver metastases: is there a possibility of cure? J Clin Oncol. 2009;27:1829-35.

19. Poston GJ, Adam R, Alberts S, Curley S, Figueras J, Haller D, et al. OncoSurge: a strategy for improving resectability with curative intent in metastatic colorectal cancer. J Clin Oncol. 2005;23:7125-34.

20. Yoshida K, Yamaguchi K, Okumura N, Tanahashi T, Kodera Y. Is conversion therapy possible in stage IV gastric cancer: the proposal of new biological categories of classification. Gastric Cancer. 2016;19:329-38.

21. Al-Agha OM, Nicastri AD. An in-depth look at Krukenberg tumor: an overview. Arch Pathol Lab Med. 2006;130:1725-30.

22. Wilke H, Muro K, Van Cutsem E, Oh SC, Bodoky G, Shimada Y, et al Ramucirumab plus paclitaxel versus placebo plus paclitaxel in patients with previously treated advanced gastric or gastro-oesophageal junction adenocarcinoma (RAINBOW): a double-blind, randomised phase 3 trial. Lancet Oncol. 2014;15:1224-35.

23. Kang YK, Boku N, Satoh T, Ryu MH, Chao Y, Kato K, et al. Nivolumab in patients with advanced gastric or gastro-oesophageal junction cancer refractory to, or intolerant of, at least two previous chemotherapy regimens (ONO-4538-12, ATTRACTION-2): a randomised, double-blind, placebo-controlled, phase 3 trial. Lancet. 2017;390:2461-71.

24. Shitara K, Doi T, Dvorkin M, Mansoor W, Arkenau HT, Prokharau A, et al. Trifluridine/tipiracil versus placebo in patients with heavily pretreated metastatic gastric cancer (TAGS): a randomised, double-blind, placebocontrolled, phase 3 trial. Lancet Oncol. 2018;19:1437-48.

25. Kodera Y, Yamamura Y, Ito S, Kanemitsu Y, Shimizu Y, Hirai T, et al. Is Borrmann type IV gastric carcinoma a surgical disease? An old problem revisited with reference to the result of peritoneal washing cytology. $J$ Surg Oncol. 2001;78:175-81.

\section{Publisher's Note}

Springer Nature remains neutral with regard to jurisdictional claims in published maps and institutional affiliations.

\section{Submit your manuscript to a SpringerOpen ${ }^{\circ}$ journal and benefit from:}

- Convenient online submission

- Rigorous peer review

- Open access: articles freely available online

- High visibility within the field

Retaining the copyright to your article

Submit your next manuscript at springeropen.com 